HWM-07-6

EMPG-07-04

hep-th/0701273

January 2007

\title{
Wilson Loops and Area-Preserving Diffeomorphisms in Twisted Noncommutative Gauge Theory
}

\author{
Mauro Riccardi and Richard J. Szabo \\ Department of Mathematics \\ and \\ Maxwell Institute for Mathematical Sciences \\ Heriot-Watt University \\ Colin Maclaurin Building, Riccarton, Edinburgh EH14 4AS, U.K. \\ mauro@ma.hw.ac.uk, R.J.Szabo@ma.hw.ac.uk
}

\begin{abstract}
We use twist deformation techniques to analyse the behaviour under area-preserving diffeomorphisms of quantum averages of Wilson loops in Yang-Mills theory on the noncommutative plane. We find that while the classical gauge theory is manifestly twist covariant, the holonomy operators break the quantum implementation of the twisted symmetry in the usual formal definition of the twisted quantum field theory. These results are deduced by analysing general criteria which guarantee twist invariance of noncommutative quantum field theories. From this a number of general results are also obtained, such as the twisted symplectic invariance of noncommutative scalar quantum field theories with polynomial interactions and the existence of a large class of holonomy operators with both twisted gauge covariance and twisted symplectic invariance.
\end{abstract}




\section{Introduction and Summary}

Noncommutative gauge theory in two dimensions possesses many interesting features that can be captured analytically and used to shed light on generic features of noncommutative field theory. It is an exactly solvable model whose partition function has been computed explicitly as a semi-classical expansion over instantons on the noncommutative torus in [39]-[41], as a sum over fluxons on the noncommutative plane in [31, 32], and also within its lattice regularization in [30, 41]. The instanton expansion has also been used to obtain exact analytic expressions for quantum averages of open Wilson lines in [11, 32, 40]. Exact Wilson loop correlators, on the other hand, have remained elusive. Perturbative computations on the noncommutative plane [3, 13] reveal that Wilson loop averages are not invariant under generic area-preserving diffeomorphisms, in marked contrast to the commutative case [50]. The quantum gauge theory itself is only invariant under the subgroup of global symplectic transformations [13] which is the largest spacetime symmetry under which the Moyal product is covariant [29]. This breaks the topological symmetry of the gauge theory making the exact semi-classical expansion of Wilson loop correlators intractable. In [22] this breakdown was interpreted nonperturbatively as the loss of covariance under gauge Morita equivalence on the noncommutative torus. Other aspects of Wilson loop correlators in two-dimensional noncommutative Yang-Mills theory have been analysed using perturbation theory in [12, 24] and numerically in [14].

In parallel developments it has been realized how to reinstate spacetime symmetries which are generically broken by noncommutativity in noncommutative field theories [6, 19, 49]. The idea, well-known from quantum group theory, is that the noncommutativity of the algebra of functions $\mathcal{A}$ should be compensated by a noncommutative deformation of the bialgebra of diffeomorphisms acting on $\mathcal{A}$. For Moyal planes this is straightforward to do as the star-product is defined in terms of an abelian Drinfeld twist element and the required deformation is a twist deformation. In this paper we will use twisted Hopf algebra techniques to investigate whether or not the twisted form of area-preserving diffeomorphims can be used to recover the full symplectic symmetry of two-dimensional noncommutative Yang-Mills theory. Along the way we will add some general insights into the properties of the much debated twisted noncommutative quantum field theories. Twisted conformal transformations in two dimensions have been analysed in [35] while generic two-dimensional twisted diffeomorphisms are discussed in [7]. Some parts of our analysis have obvious generalizations to higher-dimensional Moyal planes and the behaviour of generic noncommutative field theories under symplectomorphisms of flat spacetime $\mathbb{R}^{d}$.

The origin of twisted spacetime symmetries can be understood in a relatively straightforward way by proceeding abstractly as follows $[8,16]$. Let $\mathcal{A}=\mathcal{A}_{\theta}$ be the associative algebra over $\mathbb{C}$ generated by operators $\hat{x}^{\mu}$ obeying the Heisenberg commutation relations

$$
\left[\hat{x}^{\mu}, \hat{x}^{\nu}\right]=\mathrm{i} \theta^{\mu \nu} \text {. }
$$

Let $\hat{\partial}_{\mu} \in \operatorname{der}(\mathcal{A})$ be linear derivations of $\mathcal{A}$ defined by

$$
\left[\hat{\partial}_{\mu}, \hat{x}^{\nu}\right]=\delta_{\mu}{ }^{\nu}, \quad\left[\hat{\partial}_{\mu}, \hat{\partial}_{\nu}\right]=0 \text {. }
$$

A generic infinitesimal diffeomorphism, implemented by a vector field $\hat{\xi}=\xi^{\mu}(\hat{x}) \hat{\partial}_{\mu}$, is not an automorphism of the algebra $\mathcal{A}$ because it does not preserve the defining relations (1.1). Consider now the new operators

$$
x^{\mu} \doteq \hat{x}^{\mu}-\frac{\mathrm{i}}{2} \theta^{\mu \nu} \hat{\partial}_{\nu}, \quad \partial_{\mu} \doteq \hat{\partial}_{\mu}
$$


which extend $\mathcal{A}$ to the algebra spanned by $\operatorname{der}(\mathcal{A})$ and generate a commutative algebra

$$
\left[x^{\mu}, x^{\nu}\right]=0, \quad\left[\partial_{\mu}, x^{\nu}\right]=\delta_{\mu}^{\nu}, \quad\left[\partial_{\mu}, \partial_{\nu}\right]=0 .
$$

The meaning of the operators (1.3) is as follows. The operators $\hat{x}^{\mu}$ generate the natural action of the algebra $\mathcal{A}$ on itself via multiplication from the left, whereas $-\mathrm{i} \theta^{\mu \nu} \hat{\partial}_{\nu}$ coincide with the commuting adjoint action of $\hat{x}^{\mu}$ (using (1.1)) and generate the natural action of $\mathcal{A}$ on itself via multiplication from the right $[8]$. This right $\mathcal{A}$-module is isomorphic to the opposite algebra $\mathcal{A}^{\prime} \cong \mathcal{A}_{-\theta}$ which defines the commutant of the algebra $\mathcal{A}$ in the canonical representation on itself. One has $\mathcal{A}^{\prime} \cong J \mathcal{A} J^{-1}$ where $J$ is the canonical Tomita involution [34]. The operators (1.3) are then the generators for the action of $\mathcal{A}$ on the Morita equivalence bimodule $\mathcal{A} \otimes \mathcal{A}^{\prime}$.

The commutative bimodule algebra $\mathcal{A} \otimes \mathcal{A}^{\prime}$ carries the standard action of the diffeomorphism group, whose connected components are generated by smooth vector fields

$$
\xi=\xi^{\mu}(x) \partial_{\mu} .
$$

Substituting (1.3) into (1.5) using a formal Taylor series expansion of the smooth functions $\xi^{\mu}(x)$ gives the corresponding operators acting on $\mathcal{A}$ as

$$
\begin{aligned}
\hat{\xi} & \doteq \xi^{\mu}\left(\hat{x}-\frac{\mathrm{i}}{2} \theta \cdot \hat{\partial}\right) \hat{\partial}_{\mu} \\
& =\xi^{\mu}(\hat{x}) \hat{\partial}_{\mu}+\sum_{n=1}^{\infty}\left(-\frac{\mathrm{i}}{2}\right)^{n} \frac{1}{n !} \theta^{\mu_{1} \nu_{1}} \cdots \theta^{\mu_{n} \nu_{n}}\left(\partial_{\mu_{1}} \cdots \partial_{\mu_{n}} \xi^{\mu}\right)(\hat{x}) \hat{\partial}_{\nu_{1}} \cdots \hat{\partial}_{\nu_{n}} \hat{\partial}_{\mu} .
\end{aligned}
$$

Thus a vector field becomes a higher-order differential operator acting on the noncommutative algebra $\mathcal{A}$, and (1.6) is the usual expression for the twisted action of diffeomorphisms [6, 23, 45]. For example, the generators of the Lorentz group

$$
M^{\mu \nu}(x, \partial)=-\mathrm{i}\left(x^{\mu} \partial^{\nu}-x^{\nu} \partial^{\mu}\right)
$$

act on $\mathcal{A}$ as

$$
\hat{M}^{\mu \nu}=M^{\mu \nu}(\hat{x}, \hat{\partial})-\frac{i}{2}\left(\theta^{\mu \lambda} \hat{\partial}_{\lambda} \hat{\partial}^{\nu}-\theta^{\nu \lambda} \hat{\partial}_{\lambda} \hat{\partial}^{\mu}\right),
$$

which is the usual action of twisted Lorentz transformations [19, 49].

The extra higher order terms imply that the diffeomorphisms do not act as derivations of $\mathcal{A}$ as the Leibnitz rule is deformed. This deformation is exactly what is implemented in the twisted bialgebra of diffeomorphisms. In particular, the noncommutativity tensor $\theta^{\mu \nu}$ is in this way manifestly invariant under twisted diffeomorphisms. On fields one uses the WeylWigner correspondence [44] to implement the action of the diffeomorphism group using starproducts [6, 45]. In [8] the usual commutative action on fields is used instead, leading to a rather different class of twisted noncommutative field theories than those considered here. A novel interpretation of the Drinfeld twisting as deformed constraints in canonical quantization of noncommutative field theory can be found in [43].

When transcribed to quantum field theory, the relative simplicity of the implementation above appears to be in agreement with suggestions [27, 51] that there are no non-trivial observable consequences of the twisted Poincaré symmetry in noncommutative field theory. However, most of the debates surrounding such issues deal only with scalar fields and it is not presently 
clear what the situation is for gauge theories. To try to understand this better, in the following we will study the action of these twisted diffeomorphisms on the observables of two-dimensional noncommutative Yang-Mills theory. We will first study the action of the twisted diffeomorphism group on generic noncommutative quantum field theories in two dimensions. We will find, quite generally, that classical invariance already truncates to the subgroup of area-preserving diffeomorphisms, as expected but in a rather non-trivial way. In particular, generic scalar quantum field theories with polynomial interactions are twist invariant under symplectic transformations. When applied to gauge theory in two dimensions one can immediately infer from this the twisted symplectic invariance of the classical noncommutative Yang-Mills action and all observables. This ties in nicely with the geometric interpretation of noncommutative gauge symmetries as "deformed" symplectomorphisms of flat spacetime [34].

We point out the existence of two natural sets of holonomy observables in noncommutative gauge theory, one associated with the ordinary tensor algebra of fields and the other with the braided tensor algebra. Both classes of operators are manifestly covariant under both twisted gauge transformations [5, 48] and twisted area-preserving diffeomorphisms. The latter class defines the usual noncommutative Wilson loops which are in addition invariant under star-gauge transformations [25, 44]. Although classically invariant under twisted area-preserving diffeomorphisms, the quantum averages of these holonomies break the twisted spacetime symmetry. The twisted symmetry is not anomalous but is instead broken by the quantum implementation of Wilson loop operators in star-gauge invariant correlation functions. Thus only some nonstandard definition of the twisted quantum gauge theory can allow for full invariance under the symplectic symmetry. This provides a much more general, explicit and nonperturbative description for the loss of topological symmetry than those given in [3, 13, 22]. On the other hand, the twisted symmetry of the quantum observables under global symplectic transformations follows rather directly from the structure of the twisted bialgebra of area-preserving diffeomorphisms.

The organisation of the remainder of this paper is as follows. We begin in Section 2 with a general description of twisted symmetries and their specialization to the diffeomorphism group of spacetime, describing in particular the twisted bialgebra of area-preserving diffeomorphisms. In Section 3 we describe the generic implementation of twisted diffeomorphisms as symmetries of the quantum effective action in noncommutative field theories, showing in particular that noncommutative scalar quantum field theory is twist invariant under symplectic transformations. Finally, in Section 4 we detail the construction of a broad set of holonomy observables in twisted noncommutative gauge theory, and study in detail the behaviour of star-Wilson loops under twisted area-preserving diffeomorphisms.

\section{Twisted Spacetime Symmetries}

In this section we will discuss some basic general aspects of twisted symmetry groups of associative algebras in the context of Hopf algebras and Drinfeld twists [26, 38, 42] (see [21] for the necessary background on quantum groups). We will then specialize the discussion, following [4, 6], to the case of diffeomorphisms acting on fields on the Moyal plane. In particular, we describe the twisted bialgebra of area-preserving diffeomorphisms of $\mathbb{R}^{2}$, which will be the most pertinent example in the subsequent sections. 


\subsection{Twist Deformations of Group Actions}

Let $\mathcal{A}$ be an associative algebra over $\mathbb{C}$ with multiplication map $\mu_{0}: \mathcal{A} \otimes \mathcal{A} \rightarrow \mathcal{A}$. Let $G \subset \operatorname{Aut}(\mathcal{A})$ be a group of symmetries of $\mathcal{A}$ acting by automorphisms. Let $\mathcal{H}=\mathbb{C} G$ be the group algebra of $G$. We can make $\mathcal{H}$ into a Hopf algebra whose underlying bialgebra structure is specified by the cocommutative primitive coproduct which is the homomorphism $\Delta_{0}: \mathcal{H} \rightarrow \mathcal{H} \otimes \mathcal{H}$ defined by $\Delta_{0}(g)=g \otimes g$ on generators $g \in G$ and $\Delta_{0}(\mathrm{id})=\mathrm{id} \otimes \mathrm{id}$. The coproduct implements the action of $G$ on the tensor product $\mathcal{A} \otimes \mathcal{A}$ with the fundamental compatibility condition

$$
g \circ \mu_{0}(a \otimes b)=\mu_{0} \circ \Delta_{0}(g)(a \otimes b)
$$

for all $g \in \mathcal{H}$ and $a, b \in \mathcal{A}$. This is just the expected covariance condition on the multiplication in $\mathcal{A}$, and it is a necessary and sufficient condition for the group $G$ to act on the algebra $\mathcal{A}$.

We introduce an operator $\mathcal{F} \in \mathcal{H} \otimes \mathcal{H}$, called a twist element [26, 42], which acts on the tensor product

$$
\mathcal{F}: \mathcal{A} \otimes \mathcal{A} \longrightarrow \mathcal{A} \otimes \mathcal{A}
$$

such that the pair

$$
\left(\mathcal{A}, \mu \doteq \mu_{0} \circ \mathcal{F}\right)
$$

is still an associative algebra. If the original multiplication map $\mu_{0}$ is commutative, then generically the twisted product $\mu$ is no longer commutative. We will also write the twisted product using the star product notation $\mu(a \otimes b) \doteq a \star b$. Compatibility of the action of $G$ on the twist deformed algebra (2.3) requires twisting of the bialgebra structure on $\mathcal{H}$ as well. The condition (2.1) then becomes

$$
g \circ \mu(a \otimes b)=\mu \circ \Delta(g)(a \otimes b)
$$

for all $g \in \mathcal{H}$ and $a, b \in \mathcal{A}$. The generically non-cocommutative twisted coproduct defined by the similarity transformation

$$
\Delta: \mathcal{H} \longrightarrow \mathcal{H} \otimes \mathcal{H}, \quad \Delta \doteq \mathcal{F}^{-1} \circ \Delta_{0} \circ \mathcal{F}
$$

makes $\mathcal{H}$ into a triangular Hopf algebra. All of this requires $\mathcal{F}$ to be an invertible, co-unital two-cocycle of the Hopf algebra $\mathcal{H}$. In the star product notation we will write the left-hand side of (2.4) as $g \circ \mu(a \otimes b) \doteq g \triangleright(a \star b)$.

In many cases of interest the algebra $\mathcal{A}$ will carry generic products of arbitrary representations of the Hopf algebra $\mathcal{H}$. If an element $a \in \mathcal{A}$ transforms in a representation $R$ of the symmetry group $G$, then the corresponding action of an automorphism $g \in \mathcal{H}$ is denoted

$$
g: \mathcal{A} \longrightarrow \mathcal{A}, \quad a \longmapsto g^{(R)}(a) .
$$

On tensor products $a_{1} \otimes a_{2}$, with $a_{i} \in \mathcal{A}$ transforming in the representation $R_{i}$, the operator $g$ acts via the coproduct as

$$
g \triangleright\left(a_{1} \otimes a_{2}\right)=\Delta(g)\left(a_{1} \otimes a_{2}\right)=\left(g_{: 1}^{\left(R_{1}\right)} \otimes g_{: 2}^{\left(R_{2}\right)}\right)\left(a_{1} \otimes a_{2}\right) .
$$

Here we have used the Sweedler notation $\Delta(g)=g_{: 1} \otimes g_{: 2}$ (with a sum over $g_{: 1}$ and $g_{: 2}$ understood). 
Let us now assume that the twist leaves each factor of the tensor product $a_{1} \otimes a_{2}$ in its same representation. We can then write the compatibility condition (2.4) for $g \in G$ as

$$
\begin{aligned}
g \triangleright\left(a_{1} \star a_{2}\right) & =\mu_{0} \circ \Delta_{0}(g) \circ \mathcal{F}\left(a_{1} \otimes a_{2}\right) \\
& =\mu_{0} \circ\left(g^{\left(R_{1}\right)} \otimes g^{\left(R_{2}\right)}\right) \circ \mathcal{F}\left(a_{1} \otimes a_{2}\right) \\
& =\mu_{0} \circ\left(g^{\left(R_{1}\right)} \otimes g^{\left(R_{2}\right)}\right) \circ \mathcal{F} \circ\left(g^{\left(R_{1}\right)} \otimes g^{\left(R_{2}\right)}\right)^{-1} \circ\left(g^{\left(R_{1}\right)} \otimes g^{\left(R_{2}\right)}\right)\left(a_{1} \otimes a_{2}\right) .
\end{aligned}
$$

It follows that the group $G$ acts on the twist operator $\mathcal{F}$ itself through the similarity transformations

$$
g: \mathcal{F} \longmapsto g \triangleright \mathcal{F}=\left(g^{\left(R_{1}\right)} \otimes g^{\left(R_{2}\right)}\right) \circ \mathcal{F} \circ\left(g^{\left(R_{1}\right)} \otimes g^{\left(R_{2}\right)}\right)^{-1}
$$

In our subsequent applications we will be interested in the infinitesimal version of this construction for the case when $G$ is a continuous group of symmetries with Lie algebra $\mathfrak{g}$. In that case we take $\mathcal{H}=U(\mathfrak{g})$ to be the universal enveloping algebra of $\mathfrak{g}$, made into a Hopf algebra with bialgebra structure specified by the primitive coproduct $\Delta_{0}(X)=X \otimes$ id + id $\otimes X$ on generators $X \in \mathfrak{g}$. Then the compatibility condition (2.1) implements the usual Leibnitz rule for the action of the symmetry algebra $\mathfrak{g}$ on $\left(\mathcal{A}, \mu_{0}\right)$, while the twist deformed Leibnitz rule (2.4) allows for a representation of $\mathfrak{g}$ on (2.3). The corresponding action of the Lie algebra on the twist is given by

$$
\begin{aligned}
X: \mathcal{F} \longmapsto X \triangleright \mathcal{F} & =\left[X_{: 1}^{\left(R_{1}\right)} \otimes X_{: 2}^{\left(R_{2}\right)}, \mathcal{F}\right] \\
& =\left[X^{\left(R_{1}\right)} \otimes \mathrm{id}^{\left(R_{2}\right)}+\mathrm{id}^{\left(R_{1}\right)} \otimes X^{\left(R_{2}\right)}, \mathcal{F}\right]
\end{aligned}
$$

This machinery can also be applied if the twist element does not leave each factor of $a_{1} \otimes a_{2}$ in its own representation. In the general case we can write the compatibility condition (2.8) in a more generic way as

$$
g \triangleright\left(a_{1} \star a_{2}\right)=\mu_{0} \circ\left(g^{\left(R_{1}\right)} \otimes g^{\left(R_{2}\right)}\right) \circ \mathcal{F} \circ\left(g^{\left(L_{1}\right)} \otimes g^{\left(L_{2}\right)}\right)^{-1} \circ\left(g^{\left(L_{1}\right)} \otimes g^{\left(L_{2}\right)}\right)\left(a_{1} \otimes a_{2}\right),
$$

where the twist $\mathcal{F}$ maps the $R_{i}$ representation of $G$ into $L_{i}$. This modifies the similarity transformation (2.9) of the twist operator to

$$
g: \mathcal{F} \longmapsto g \triangleright \mathcal{F}=\left(g^{\left(R_{1}\right)} \otimes g^{\left(R_{2}\right)}\right) \circ \mathcal{F} \circ\left(g^{\left(L_{1}\right)} \otimes g^{\left(L_{2}\right)}\right)^{-1} .
$$

\subsection{Braided Tensor Calculus}

The generic situation of interest for noncommutative field theory is when $\mathcal{A}$ is taken to be the algebra of smooth functions on a manifold $\mathcal{M}$ (with appropriate boundary conditions when $\mathcal{M}$ is non-compact), with pointwise multiplication $\mu_{0}$, and with $G=\operatorname{Diff}(\mathcal{M})$ the group of diffeomorphisms of $\mathcal{M}$. In this case the algebra $\mathcal{A}$ will contain arbitrary products of representations of the corresponding Hopf algebra $\mathcal{H}$ determined by tensorial rank, and one is in the situation described in Section 2.1 above. In this paper we are primarily interested in the case where $\mathcal{M}=\mathbb{R}^{2}$ and the twist element is the bidifferential operator

$$
\mathcal{F}=\exp \left(\frac{\mathrm{i} \theta}{2}\left(\partial_{1} \otimes \partial_{2}-\partial_{2} \otimes \partial_{1}\right)\right)=\sum_{k=0}^{\infty} \frac{(\mathrm{i} \theta / 2)^{k}}{k !}\left(\partial_{1} \otimes \partial_{2}-\partial_{2} \otimes \partial_{1}\right)^{k}
$$


with $\theta \in \mathbb{R}$ constant. This defines an abelian Drinfeld twist and the corresponding star product is the Moyal product on the algebra of functions on $\mathbb{R}^{2}$. Many of the following results hold analogously for higher-dimensional Moyal planes.

The connected components of the diffeomorphism group are generated by smooth vector fields $\xi=\xi^{\mu}(x) \partial_{\mu}$. Their infinitesimal action on $\mathcal{A}$ is denoted $\delta_{\xi}$, and the elements of $\mathcal{H}$ act on $\mathcal{A}$ by the natural extension of the Lie derivative. Since the twist operator (2.13) is constructed from translation generators, i.e. constant diffeomorphisms, each factor of any tensor product $f_{1} \otimes f_{2}$ of two fields remains in its own representation under the action of $\mathcal{F}$. This follows from the identity

$$
\left[\partial_{\mu}, \delta_{\xi}\right]=\delta_{\partial_{\mu} \xi}
$$

Then the transformation (2.10) of the twist in the case at hand reads

$$
\begin{aligned}
\delta_{\xi} \triangleright \mathcal{F} & =-\mathcal{F} \circ \sum_{k=1}^{\infty} \frac{(-\mathrm{i} \theta / 2)^{k}}{k !} \overbrace{\left[\partial_{1} \otimes \partial_{2}-\partial_{2} \otimes \partial_{1}, \ldots,\left[\partial_{1} \otimes \partial_{2}-\partial_{2} \otimes \partial_{1}\right.\right.}^{k}, \Delta_{0}\left(\delta_{\xi}\right)] \ldots] \\
& =-\mathcal{F} \circ \sum_{k=1}^{\infty} \frac{(-\mathrm{i} \theta / 2)^{k}}{k !} \sum_{l=0}^{k}\left(\begin{array}{c}
k \\
l
\end{array}\right)(-1)^{l}\left(\delta_{\partial_{1}^{k-l} \partial_{2}^{l} \xi}^{\left(R_{1}\right)} \otimes \partial_{2}^{k-l} \partial_{1}^{l}+(-1)^{k} \partial_{2}^{k-l} \partial_{1}^{l} \otimes \delta_{\partial_{1}^{k-l} \partial_{2}^{l} \xi}^{\left(R_{2}\right)}\right) .
\end{aligned}
$$

It follows that the transformation of the star product of two fields under an infinitesimal diffeomorphism of $\mathbb{R}^{2}$ is given by

$$
\begin{aligned}
\delta_{\xi} \triangleright\left(f_{1} \star f_{2}\right)=-\mu\left(\sum_{k=0}^{\infty} \frac{(-\mathrm{i} \theta / 2)^{k}}{k !} \sum_{l=0}^{k}\left(\begin{array}{l}
k \\
l
\end{array}\right)(-1)^{l}\right. & \left(\delta_{\partial_{1}^{k-l} \partial_{2}^{l} \xi}^{\left(R_{1}\right)} f_{1} \otimes \partial_{2}^{k-l} \partial_{1}^{l} f_{2}\right. \\
& \left.\left.+(-1)^{k} \partial_{2}^{k-l} \partial_{1}^{l} f_{1} \otimes \delta_{\partial_{1}^{k-l} \partial_{2}^{l} \xi}^{\left(R_{2}\right)} f_{2}\right)\right) .
\end{aligned}
$$

Let us work out some examples of the transformation rule (2.16) which will be used in the sequel. If both $f_{1}$ and $f_{2}$ are scalar fields in $\mathcal{A}$, then $\delta_{\xi}^{\left(R_{1}\right)}=\delta_{\xi}^{\left(R_{2}\right)}$ is the differential operator $-\xi^{\mu} \partial_{\mu}$ and one has

$$
\begin{aligned}
\delta_{\xi} \triangleright\left(f_{1} \star f_{2}\right)=-\mu\left(\sum_{k=0}^{\infty} \frac{(-\mathrm{i} \theta / 2)^{k}}{k !} \sum_{l=0}^{k}\left(\begin{array}{l}
k \\
l
\end{array}\right)\right. & (-1)^{l}\left(\left(\partial_{1}^{k-l} \partial_{2}^{l} \xi^{\mu}\right) \partial_{\mu} f_{1} \otimes \partial_{2}^{k-l} \partial_{1}^{l} f_{2}\right. \\
& \left.\left.+(-1)^{k} \partial_{2}^{k-l} \partial_{1}^{l} f_{1} \otimes\left(\partial_{1}^{k-l} \partial_{2}^{l} \xi^{\mu}\right) \partial_{\mu} f_{2}\right)\right) .
\end{aligned}
$$

This can be seen to be equal to the expression

$$
\begin{aligned}
& \mu_{0}\left(-\left(\xi^{\mu} \partial_{\mu} \otimes \mathrm{id}+\mathrm{id} \otimes \xi^{\mu} \partial_{\mu}\right) \circ \mathcal{F}\left(f_{1} \otimes f_{2}\right)\right) \\
& =-\sum_{k=0}^{\infty} \frac{(\mathrm{i} \theta / 2)^{k}}{k !} \sum_{l=0}^{k}\left(\begin{array}{l}
k \\
l
\end{array}\right)(-1)^{l}\left(\left(\xi^{\mu} \partial_{1}^{k-l} \partial_{2}^{l} \partial_{\mu} f_{1}\right)\left(\partial_{2}^{k-l} \partial_{1}^{l} f_{2}\right)\right. \\
& \left.+(-1)^{k}\left(\partial_{2}^{k-l} \partial_{1}^{l} f_{1}\right)\left(\xi^{\mu} \partial_{1}^{k-l} \partial_{2}^{l} \partial_{\mu} f_{2}\right)\right) .
\end{aligned}
$$

Hence if both $f_{1}$ and $f_{2}$ are scalar fields, then their star-product $f_{1} \star f_{2}$ also transforms as a scalar field,

$$
\delta_{\xi} \triangleright\left(f_{1} \star f_{2}\right)=-\mu_{0}\left(\left(\xi^{\mu} \partial_{\mu} \otimes \mathrm{id}+\mathrm{id} \otimes \xi^{\mu} \partial_{\mu}\right) \circ \mathcal{F}\left(f_{1} \otimes f_{2}\right)\right)=-\xi^{\mu} \partial_{\mu}\left(f_{1} \star f_{2}\right) .
$$


If instead we take the product of two tensor fields, then we have to account for the transformations with respect to contractions of the indices as well. From (2.16) we can work out as an example the case of two rank one tensor fields $f_{1}=V_{\mu}$ and $f_{2}=W^{\nu}$. Since

$$
\begin{aligned}
\delta_{\xi}^{\left(R_{1}\right)} V_{\mu} & =-\xi^{\sigma} \partial_{\sigma} V_{\mu}-\left(\partial_{\mu} \xi^{\sigma}\right) V_{\sigma}, \\
\delta_{\xi}^{\left(R_{2}\right)} W^{\mu} & =-\xi^{\sigma} \partial_{\sigma} W^{\mu}+\left(\partial_{\sigma} \xi^{\mu}\right) W^{\sigma},
\end{aligned}
$$

we easily find that

$$
\begin{aligned}
& \delta_{\xi} \triangleright\left(V_{\mu} \star W^{\nu}\right)=-\xi^{\sigma} \partial_{\sigma}\left(V_{\mu} \star W^{\nu}\right) \\
& +\mu\left(\sum _ { n = 0 } ^ { \infty } \frac { ( - \mathrm { i } \theta / 2 ) ^ { n } } { n ! } \sum _ { l = 0 } ^ { n } ( \begin{array} { l } 
{ n } \\
{ l }
\end{array} ) ( - 1 ) ^ { l } \left[\left(-\left(\partial_{1}^{n-l} \partial_{2}^{l} \partial_{\mu} \xi^{\sigma}\right) V_{\sigma}\right) \otimes\left(\partial_{2}^{n-l} \partial_{1}^{l} W^{\nu}\right)\right.\right. \\
& \left.\left.+\left(\partial_{2}^{n-l} \partial_{1}^{l} V_{\mu}\right) \otimes\left(\left(\partial_{1}^{n-l} \partial_{2}^{l} \partial_{\sigma} \xi^{\mu}\right) W^{\sigma}\right)\right]\right) \\
& =-\xi^{\sigma} \partial_{\sigma}\left(V_{\mu} \star W^{\nu}\right) \\
& +\mu_{0}\left(\mathcal{F} \circ\left(V_{\sigma} \otimes 1\right) \circ \mathcal{F}^{-1}\left(-\partial_{\mu} \xi^{\sigma} \otimes W^{\nu}\right)+\mathcal{F} \circ\left(1 \otimes W^{\sigma}\right) \circ \mathcal{F}^{-1}\left(V_{\mu} \otimes-\partial_{\sigma} \xi^{\nu}\right)\right) .
\end{aligned}
$$

Using the similarity transformations

$$
\begin{gathered}
\mathcal{F} \circ\left(V_{\sigma} \otimes 1\right) \circ \mathcal{F}^{-1}=\sum_{n=1}^{\infty} \frac{(\mathrm{i} \theta / 2)^{n}}{n !} \sum_{l=0}^{n}\left(\begin{array}{c}
n \\
l
\end{array}\right)(-1)^{l}\left(\partial_{1}^{n-l} \partial_{2}^{l} V_{\sigma}\right) \otimes \partial_{2}^{n-l} \partial_{1}^{l}, \\
\mathcal{F} \circ\left(1 \otimes W^{\sigma}\right) \circ \mathcal{F}^{-1}=\sum_{n=0}^{\infty} \frac{(\mathrm{i} \theta / 2)^{n}}{n !} \sum_{l=0}^{n}\left(\begin{array}{c}
n \\
l
\end{array}\right)(-1)^{l} \partial_{1}^{n-l} \partial_{2}^{l} \otimes\left(\partial_{2}^{n-l} \partial_{1}^{l} W^{\sigma}\right),
\end{gathered}
$$

we obtain

$$
\begin{aligned}
\delta_{\xi} \triangleright\left(V_{\mu} \star W^{\nu}\right)= & -\xi^{\sigma} \partial_{\sigma}\left(V_{\mu} \star W^{\nu}\right) \\
& +\mu_{0}\left(\left(-\partial_{\mu} \xi^{\sigma} \otimes 1\right) \circ \mathcal{F}\left(V_{\sigma} \otimes W^{\nu}\right)\right)+\mu_{0}\left(\left(1 \otimes \partial_{\sigma} \xi^{\nu}\right) \circ \mathcal{F}\left(V_{\mu} \otimes W^{\sigma}\right)\right)
\end{aligned}
$$

which is the usual rule of tensor calculus for the transformation of a tensor field of rank $(1,1)$.

In particular, the contraction $V_{\mu} \star W^{\mu}$ transforms expectedly as a scalar field,

$$
\begin{aligned}
\delta_{\xi} \triangleright\left(V_{\mu} \star W^{\mu}\right) & =-\xi^{\sigma} \partial_{\sigma}\left(V_{\mu} \star W^{\mu}\right)+\mu_{0}\left(\left(1 \otimes \partial_{\mu} \xi^{\sigma}-\partial_{\mu} \xi^{\sigma} \otimes 1\right) \circ \mathcal{F}\left(V_{\sigma} \otimes W^{\mu}\right)\right) \\
& =-\xi^{\sigma} \partial_{\sigma}\left(V_{\mu} \star W^{\mu}\right)
\end{aligned}
$$

because in the second term the derivative operators of $\mathcal{F}$ do not act on $\xi$. Note that if the vector field $\xi$ generates a linear affine transformation in $\mathfrak{g l}(2, \mathbb{R}) \rtimes \mathbb{R}^{2}$, given by

$$
\xi^{\sigma}(x)=L_{\mu}^{\sigma} x^{\mu}+a^{\sigma}
$$

with constant tensors $L_{\mu}^{\sigma}$ and $a^{\sigma}$, then one has

$$
1 \otimes \partial_{\mu} \xi^{\sigma}=L_{\mu}^{\sigma}(1 \otimes 1)=\partial_{\mu} \xi^{\sigma} \otimes 1
$$

This automatically guarantees the scalar property of the contraction (2.24) under global diffeomorphisms. 
The general result [4, 6] is that covariant expressions in ordinary (untwisted, commutative) tensor calculus are still covariant after the twist deformation. In Section 4 we will use this feature to infer the classical invariance of two-dimensional noncommutative Yang-Mills theory under twisted area-preserving diffeomorphisms. Note that since the compatibility equation (2.4) is equivalent to the untwisted one in (2.1), the tensor calculus constructed by using the standard pointwise multiplication $\mu_{0}$ is still covariant as well under untwisted diffeomorphisms.

\subsection{Twisted Area-Preserving Diffeomorphisms}

In order to gain a better understanding of the formalism above, we now display explicitly the twisted bialgebra structure of the Lie algebra of area-preserving diffeomorphisms of $\mathbb{R}^{2}$, which will be our prominent example of a twisted symmetry in this paper. These are the symplectic transformations preserving the area form $\mathrm{d}^{2} x=\mathrm{d} x^{1} \wedge \mathrm{d} x^{2}$. Let us denote the generators by $\mathfrak{L}_{m, n}, m, n \in \mathbb{N}$. We select the representation on $\mathcal{A}$ given by the differential operators

$$
\mathfrak{L}_{n, m}=m x_{1}^{n} x_{2}^{m-1} \partial_{1}-n x_{1}^{n-1} x_{2}^{m} \partial_{2} .
$$

The elementary Lie brackets are those of the infinite-dimensional $W_{1+\infty}$ algebra

$$
\left[\mathfrak{L}_{m, n}, \mathfrak{L}_{p, q}\right]=(n p-m q) \mathfrak{L}_{m+p-1, n+q-1} .
$$

Then the twisted coproduct of these generators can be straightforwardly worked out to be

$$
\begin{array}{r}
\Delta\left(\mathfrak{L}_{n, m}\right)=\mathfrak{L}_{n, m} \otimes 1+1 \otimes \mathfrak{L}_{n, m} \\
\quad+\sum_{k=1}^{n+m-1}(-\mathrm{i} \theta / 2)^{k} \sum_{l=0}^{k}\left(\begin{array}{c}
n \\
k-l
\end{array}\right)\left(\begin{array}{c}
m \\
l
\end{array}\right)(-1)^{l}\left[(-1)^{k} \mathfrak{L}_{n-k+l, m-l} \otimes\left(\partial_{1}^{k-l} \partial_{2}^{l}\right)\right. \\
\left.+\left(\partial_{1}^{k-l} \partial_{2}^{l}\right) \otimes \mathfrak{L}_{n-k+l, m-l}\right] .
\end{array}
$$

In particular, for the generators $\mathfrak{L}_{m, n}$ with $n+m \leq 2$ one finds

$$
\begin{aligned}
& \Delta\left(\mathfrak{L}_{0,1}\right)=\Delta\left(\partial_{1}\right)=1 \otimes \partial_{1}+\partial_{1} \otimes 1, \\
& \Delta\left(\mathfrak{L}_{1,0}\right)=\Delta\left(-\partial_{2}\right)=1 \otimes\left(-\partial_{2}\right)+\left(-\partial_{2}\right) \otimes 1, \\
& \Delta\left(\mathfrak{L}_{1,1}\right)=\Delta\left(x_{1} \partial_{1}-x_{2} \partial_{2}\right)=1 \otimes\left(x_{1} \partial_{1}-x_{2} \partial_{2}\right)+\left(x_{1} \partial_{1}-x_{2} \partial_{2}\right) \otimes 1, \\
& \Delta\left(\mathfrak{L}_{2,0}\right)=\Delta\left(-2 x_{1} \partial_{2}\right)=1 \otimes\left(-2 x_{1} \partial_{2}\right)+\left(-2 x_{1} \partial_{2}\right) \otimes 1, \\
& \Delta\left(\mathfrak{L}_{0,2}\right)=\Delta\left(2 x_{2} \partial_{1}\right)=1 \otimes\left(2 x_{2} \partial_{1}\right)+\left(2 x_{2} \partial_{1}\right) \otimes 1 .
\end{aligned}
$$

Thus these operators are all primitive with respect to the twisted coproduct $\Delta$, and hence the tensor products of their representation is unchanged. They generate a subalgebra of $W_{1+\infty}$ isomorphic to $\mathfrak{s l}(2, \mathbb{R}) \rtimes \mathbb{R}^{2}$, comprising global diffeomorphisms which generate linear unimodular affine transformations with $L^{\mu}{ }_{\mu}=0$ in (2.25). We can further easily check from (2.30) that these are the only generators whose primitive coproducts are unchanged by the twist, since higher order terms in $\theta$ can only cancel when simultaneously $k \leq 1$ and $k-l \leq 1$, due to the fact that the generators $\mathfrak{L}_{m, n}$ are linear in derivative operators. Therefore the Lie algebra $\mathfrak{s l}(2, \mathbb{R}) \rtimes \mathbb{R}^{2}$ of global area-preserving diffeomorphisms is the largest subalgebra of $W_{1+\infty}$ whose representations are unaffected by the twist. This fact will be crucial in determining under which symmetry the physical quantum observables of noncommutative Yang-Mills theory have twisted invariance. It provides an alternative way to understand the known covariance of the Moyal product [29] and of noncommutative gauge theory [13] under linear affine transformations. 


\section{Spacetime Symmetries of Twisted Noncommutative Field Theory}

In this section we will perform a general analysis of the invariance of two-dimensional noncommutative field theory under the twisted spacetime symmetries of the previous section. We adapt the point of view that the twist deformation realizes diffeomorphisms as potential internal symmetries of noncommutative field theory [34, 44, so that some of the ensuing statements also apply to other classes of twisted symmetries. We will find that, generically, already at the classical level the twisted invariance truncates to the area-preserving diffeomorphisms of Section 2.3. However, this truncation does not arise from setting the Jacobian of a map equal to one in order to preserve the area form $\mathrm{d}^{2} x$ in the action, as the twist does not act on any integration measure. At the quantum level, we will interpret the potential loss of twisted symmetry as anomalous behaviour of the field theory. In the next section these general considerations will be applied to Wilson loop correlators in noncommutative Yang-Mills theory.

\subsection{Symmetries and Ward Identities}

We begin by recalling how to implement (spacetime) symmetries in quantum field theory. Consider a (noncommutative) field theory on $\mathbb{R}^{2}$ with fields $\Phi_{i}$ and action functional $S[\Phi]$. The symbol $\Phi_{i}$ in general collectively denotes all fundamental fields such as matter and gauge fields, as well as any auxilliary and ghost fields, and $S[\Phi]$ may generally include Lagrange multiplier and gauge-fixing terms. Under a symmetry transformation of the field theory (for instance a diffeomorphism of spacetime), the infinitesimal variation of the fields is denoted

$$
\Phi_{i} \longmapsto \Phi_{i}+\delta \Phi_{i}
$$

We wish to implement this transformation as a symmetry of the quantum correlation functions of a set of operators $\mathcal{O}_{1}(\Phi), \ldots, \mathcal{O}_{n}(\Phi)$ in the quantum field theory. In path integral quantization, this roughly leads to the identification

$$
\int \mathcal{D} \Phi \mathrm{e}^{-S[\Phi]} \mathcal{O}_{1}(\Phi) \cdots \mathcal{O}_{n}(\Phi)=\int \mathcal{D}(\Phi+\delta \Phi) \mathrm{e}^{-S[\Phi+\delta \Phi]} \mathcal{O}_{1}(\Phi+\delta \Phi) \cdots \mathcal{O}_{n}(\Phi+\delta \Phi)
$$

or equivalently to first order in $\delta \Phi_{i}$ one has the Schwinger-Dyson equations

$$
\int \mathcal{D} \Phi \frac{\delta}{\delta \Phi_{i}}\left(\mathrm{e}^{-S[\Phi]} \mathcal{O}_{1}(\Phi) \cdots \mathcal{O}_{n}(\Phi)\right)=0
$$

One issue is whether or not the functional integration, or the path integral measure, gives some additional contributions to these expressions. In standard quantum field theory parlance, this is the same as the question of whether or not there is some form of an anomaly in the quantum theory.

To investigate this problem more precisely, we couple the fields $\Phi_{i}$ to external source fields $J^{i}$ through the pairing $(J, \Phi) \doteq \int \mathrm{d}^{2} x J^{i} \Phi_{i}$. The response of the system to these external sources is encoded in the generating functional for connected Green's functions given by

$$
\mathcal{W}[J]=-\log \int \mathcal{D} \Phi \mathrm{e}^{-S[\Phi]-(J, \Phi)}
$$


The effective action is defined by the Legendre transform

$$
\Gamma[\hat{\Phi}] \doteq\left(J^{i} \hat{\Phi}_{i}-\mathcal{W}[J]\right)_{\frac{\delta \mathcal{W}[J]}{\delta J^{i}}=\hat{\Phi}_{i}} .
$$

Under the transformation (3.1), we make the natural assumption that the functional integration measure is invariant, or equivalently that in the path integral one can identify

$$
\mathcal{D}(\Phi+\delta \Phi) \mathrm{e}^{-S[\Phi+\delta \Phi]}=\mathcal{D} \Phi \mathrm{e}^{-S[\Phi]} .
$$

Then we obtain the fundamental Ward identity

$$
0=\int \mathrm{d}^{2} x \frac{\delta \Gamma[\hat{\Phi}]}{\delta \hat{\Phi}_{i}} \frac{\left\langle 0\left|\delta \hat{\Phi}_{i}\right| 0\right\rangle_{J_{\hat{\Phi}_{i}}}}{\langle 0 \mid 0\rangle_{\hat{\Phi}_{i}}}
$$

where

$$
J_{\hat{\Phi}_{i}}=-\frac{\delta \Gamma[\hat{\Phi}]}{\delta \hat{\Phi}_{i}}
$$

and the vacuum expectation values are taken in the original quantum field theory coupled to the sources $J_{\hat{\Phi}_{i}}$. The Ward identity (3.7) is a statement of the symmetry of the quantum effective action. These statements all implicitly assume that an appropriate regularization of the Green's functions has been specified which respects the symmetry (3.1).

\subsection{Implementation of Twisted Symmetries}

Let us now examine what features of the above analysis can be extended to the case where the variation (3.1) represents twisted symmetries of a noncommutative field theory, and in particular twisted diffeomorphisms. In implementing these identities, crucial use is made of the Leibnitz rule for the variational (or the functional derivative) operator. In the twisted field theory the Leibnitz rule is also twisted, giving an additional contribution to the variations of star-products of fields, or equivalently of the twist element $\mathcal{F}$ as in (2.15). If anomalous behaviour arises as described above, then the hidden symmetry represented by twisted diffeomorphisms cannot be implemented at the quantum level. We will begin by deducing the most general possible twisted spacetime symmetry of noncommutative field theory.

Let us start with a simple, explicit example of a noncommutative field theory for illustration. Consider a real scalar field theory with action of the form

$$
S_{V}[\phi]=\frac{1}{2} \int \mathrm{d}^{2} x\left(\phi \square \phi+m^{2} \phi^{2}+2 V(\phi)\right) .
$$

Let us assume, as is customary in noncommutative field theory, that the path integral measure is the standard Feynman measure $\mathcal{D} \phi=\prod_{x \in \mathbb{R}^{2}} \mathrm{~d} \phi(x)$ of commutative quantum field theory. This definition guarantees that when $V=0$ the generating functional (3.4) is given by $\mathcal{W}[J]=(J, \mathcal{C} J)$, where $\mathcal{C}$ is the free propagator of the quantum field theory. It also agrees in this case with the twist-covariant functional integral constructed in [33]. Then the identification (3.6) holds, and so we need only study the twisted transformation of the action (3.9) under a shift of the field $\phi$ by an infinitesimal diffeomorphism $\delta_{\xi} \phi$. For this, we use the twisted covariance of the star-product (2.16) as explained in Section 2.2, 
To deduce the general structure of the variation, consider first noncommutative $\phi^{3}$-theory with interaction potential $V(\phi)=g \phi \star \phi \star \phi$. One then finds

$$
\begin{aligned}
S_{\phi^{3}}[\phi] \longmapsto & S_{\phi^{3}}[\phi]-g \int \mathrm{d}^{2} x \mu\left(\mu_{0}\left(\delta_{\xi} \triangleright \mathcal{F}(\phi \otimes \phi)\right) \otimes \phi\right)-g \int \mathrm{d}^{2} x \mu\left(\phi \otimes \mu_{0}\left(\delta_{\xi} \triangleright \mathcal{F}(\phi \otimes \phi)\right)\right) \\
& =S[\phi]-2 g \int \mathrm{d}^{2} x \mu_{0}\left(\delta_{\xi} \triangleright \mathcal{F}(\phi \otimes \phi)\right) \star \phi,
\end{aligned}
$$

where we have used the trace property

$$
\int \mathrm{d}^{2} x f_{1} \star f_{2}=\int \mathrm{d}^{2} x f_{1} f_{2}=\int \mathrm{d}^{2} x f_{2} \star f_{1}
$$

for any two Schwartz fields $f_{1}, f_{2} \in \mathcal{A}$. For noncommutative $\phi^{4}$-theory with interaction potential $V(\phi)=g \phi \star \phi \star \phi \star \phi$, one instead has

$$
\begin{aligned}
S_{\phi^{4}}[\phi] \longmapsto S_{\phi^{4}}[\phi] & -g \int \mathrm{d}^{2} x \mu\left[\mu\left(\mu_{0}\left(\delta_{\xi} \triangleright \mathcal{F}(\phi \otimes \phi)\right) \otimes \phi\right) \otimes \phi\right] \\
& -g \int \mathrm{d}^{2} x \mu\left[\mu\left(\phi \otimes \mu_{0}\left(\delta_{\xi} \triangleright \mathcal{F}(\phi \otimes \phi)\right)\right) \otimes \phi\right] \\
& -g \int \mathrm{d}^{2} x \mu\left(\mu(\phi \otimes \phi) \otimes \mu_{0}\left(\delta_{\xi} \triangleright \mathcal{F}(\phi \otimes \phi)\right)\right) \\
=S[\phi] & -3 g \int \mathrm{d}^{2} x \mu_{0}\left(\delta_{\xi} \triangleright \mathcal{F}(\phi \otimes \phi)\right) \star \phi \star \phi .
\end{aligned}
$$

These formulas generalize to arbitrary polynomial interactions in the obvious way.

We see that the general structure of the variational terms that we need to manipulate are of the form

$$
\begin{array}{r}
\int \mathrm{d}^{2} x \mu_{0}\left(\delta_{\xi} \triangleright \mathcal{F}\left(f_{1} \otimes f_{2}\right)\right)=-\int \mathrm{d}^{2} x \sum_{k=1}^{\infty} \frac{(-\mathrm{i} \theta / 2)^{k}}{k !} \sum_{l=0}^{k}\left(\begin{array}{l}
k \\
l
\end{array}\right)(-1)^{l}\left[\left(\delta_{\partial_{1}^{k-l} \partial_{2}^{l} \xi}^{\left(R_{1}\right)} f_{1}\right)\left(\partial_{2}^{k-l} \partial_{1}^{l} f_{2}\right)\right. \\
\left.+(-1)^{k}\left(\partial_{2}^{k-l} \partial_{1}^{l} f_{1}\right)\left(\delta_{\partial_{1}^{k-l} \partial_{2}^{l} \xi}^{\left(R_{2}\right)} f_{2}\right)\right] .
\end{array}
$$

When $f_{1}$ and $f_{2}$ are scalar Schwartz fields on $\mathbb{R}^{2}$, after integrating by parts this becomes

$$
\begin{gathered}
\int \mathrm{d}^{2} x \mu_{0}\left(\delta_{\xi} \triangleright \mathcal{F}\left(f_{1} \otimes f_{2}\right)\right) \\
=-\int \mathrm{d}^{2} x \sum_{k=1}^{\infty} \frac{(-\mathrm{i} \theta / 2)^{k}}{k !} \sum_{l=0}^{k}\left(\begin{array}{l}
k \\
l
\end{array}\right)(-1)^{l}\left[\begin{array}{c}
\left(\partial_{1}^{k-l} \partial_{2}^{l} \xi^{\mu}\right)\left(\partial_{2}^{k-l} \partial_{1}^{l} f_{2}\right)\left(\partial_{\mu} f_{1}\right) \\
\left.+(-1)^{k}\left(\partial_{2}^{k-l} \partial_{1}^{l} f_{1}\right)\left(\partial_{1}^{k-l} \partial_{2}^{l} \xi^{\mu}\right)\left(\partial_{\mu} f_{2}\right)\right] \\
=-\int \mathrm{d}^{2} x \sum_{k=1}^{\infty} \frac{(\mathrm{i} \theta / 2)^{k}}{k !} \sum_{l=0}^{k}\left(\begin{array}{l}
k \\
l
\end{array}\right)(-1)^{l} \xi^{\mu} \partial_{\mu}\left[\left(\partial_{1}^{k-l} \partial_{2}^{l} f_{1}\right)\left(\partial_{2}^{k-l} \partial_{1}^{l} f_{2}\right)\right. \\
\left.+\left(\partial_{1}^{k-l} \partial_{2}^{l} f_{1}\right)\left(\partial_{2}^{k-l} \partial_{1}^{l} f_{2}\right)\right] .
\end{array}\right.
\end{gathered}
$$


After another integration by parts, the integral vanishes when

$$
\operatorname{div}(\xi)=\partial_{\mu} \xi^{\mu}=0
$$

This is a necessary and sufficient condition for the vector field $\xi$ to generate an infinitesimal areapreserving diffeomorphism of $\mathbb{R}^{2}$. It follows that noncommutative scalar field theory possesses twisted symplectic symmetry.

This calculation can be extended to cases in which $f_{1}$ and $f_{2}$ are not scalar fields. Consider as an example the contraction $V_{\sigma} \star W^{\sigma}$. Then the part of (3.13) corresponding to the transformations with respect to contractions of the indices is given by the integral

$$
\begin{gathered}
-\int \mathrm{d}^{2} x \sum_{k=1}^{\infty} \frac{(-\mathrm{i} \theta / 2)^{k}}{k !} \sum_{l=0}^{k}\left(\begin{array}{l}
k \\
l
\end{array}\right)(-1)^{l}\left[\left(\partial_{1}^{k-l} \partial_{2}^{l} \partial_{\mu} \xi^{\sigma}\right)\left(\partial_{2}^{k-l} \partial_{1}^{l} V_{\sigma}\right) W^{\mu}\right. \\
\left.-(-1)^{k}\left(\partial_{2}^{k-l} \partial_{1}^{l} W^{\sigma}\right)\left(\partial_{1}^{k-l} \partial_{2}^{l} \partial_{\sigma} \xi^{\mu}\right) V_{\mu}\right] \\
=-\int \mathrm{d}^{2} x \sum_{k=1}^{\infty} \frac{(\mathrm{i} \theta / 2)^{k}}{k !} \sum_{l=0}^{k}\left(\begin{array}{c}
k \\
l
\end{array}\right)(-1)^{l}\left[\partial_{\mu} \xi^{\sigma}\left(\partial_{1}^{k-l} \partial_{2}^{l} W^{\mu}\right)\left(\partial_{2}^{k-l} \partial_{1}^{l} V_{\sigma}\right)\right. \\
\left.-\partial_{\sigma} \xi^{\mu}\left(\partial_{1}^{k-l} \partial_{2}^{l} W^{\sigma}\right)\left(\partial_{2}^{k-l} \partial_{1}^{l} V_{\mu}\right)\right] .
\end{gathered}
$$

The integral trivially vanishes after relabelling indices. This purely algebraic fact is linked to the twisted covariance of the classical field theory that we established in Section 2.2. and it enables us to extend the classical symplectic symmetry to twisted noncommutative field theories involving fields of higher rank.

Thus given any noncommutative field theory which is classically twist invariant under areapreserving diffeomorphisms, one can still write down, modulo boundary contributions, the Ward identity (3.7) for the effective action $\Gamma$ with $\delta \Phi_{i}$ an area-preserving diffeomorphism. This is just the statement that the quantum effective action is twist invariant under symplectic transformations. Recall that this holds only if the condition (3.6) is satisfied. The twist invariance of the standard Feynman path integral measure $\mathcal{D} \Phi$ has been somewhat of a matter of debate in the literature [9, 47, 51]. We will not enter into this debate nor elaborate here on the construction of the functional integral which defines the twisted quantum field theory [33, 37]. Our main analysis of the Wilson loop in the next section will be independent of this definition in any case.

\section{Wilson Loops in Twisted Noncommutative Gauge Theory}

In this final section we will apply the formalism developed thus far to investigate the twisted symmetries of Wilson loop averages in two-dimensional noncommutative Yang-Mills theory. We will first describe the construction of Wilson loop operators in the twisted quantum gauge theory, and then proceed to analyse the correlators. We will find that the correlation functions of the usual noncommutative Wilson loop operators are not twist invariant under area-preserving diffeomorphisms of $\mathbb{R}^{2}$. Contrary to the untwisted case, where even the classical gauge theory is only invariant under the global $\mathfrak{s l}(2, \mathbb{R}) \rtimes \mathbb{R}^{2}$ subalgebra described in Section 2.3 [13], the Wilson loop operators on their own break the full classical $W_{1+\infty}$ symmetry of the twisted gauge theory 
at the quantum level (up to global symplectic transformations). This provides a rather more general, explicit and nonperturbative description for this loss of invariance than those given previously in [3, 13, 22].

\subsection{Wilson Loop Operators}

Wilson loops are defined in terms of the holonomy operator

$$
\mathrm{hol}^{(R)}: \Omega \mathcal{M} \longrightarrow G^{(R)},
$$

where $\Omega \mathcal{M}$ is the loop space of $\mathcal{M}=\mathbb{R}^{2}, G$ is the gauge group, and $R$ is an irreducible unitary representation of $G$. Let us examine the effect of the twist deformation on this operator. The twist changes the way in which the group $G$ (and therefore hol ${ }^{(R)}(\gamma)$ for each $\gamma \in \Omega \mathcal{M}$ ) acts on tensor products of fields, but not the group structure itself. This implies that the composition law on the group of gauge transformations is the ordinary pointwise one induced by the multiplication in $G$, i.e. if $u_{1}, u_{2}: \mathcal{M} \rightarrow G$ are gauge transformations, then $u_{1} \circ u_{2} \doteq u_{1} u_{2}$. In particular, there is no restriction on the allowed gauge groups for which the twist deformation can be implemented [5, 48]. This is radically different from the usual definition in noncommutative gauge theory [25, 44], and has also been a focal point of much debate in the literature [2, 17, 18,

Let us regard a given loop $\gamma \in \Omega \mathcal{M}$ as a smooth embedding

$$
\gamma:[0,1] \longrightarrow \mathcal{M}, \quad s \longmapsto z(s)
$$

with $z(0)=z(1)$. Let $A$ be a $\mathfrak{g}$-valued connection one-form on $\mathcal{M}$. Then we can define the holonomy operator infinitesimally along $\gamma$ as

$$
\operatorname{hol}^{(R)}(\gamma)=\prod_{z \in \gamma}^{\mathcal{P}}\left(\mathrm{id}^{(R)}+\mathrm{i} \mathrm{d} z^{\mu} A_{\mu}^{(R)}(z)\right),
$$

where the superscript $\mathcal{P}$ denotes path ordering along the loop $\gamma$. In this setting it naturally corresponds to the solution of the parallel transport equation

$$
\frac{\partial \mathrm{hol}^{(R)}}{\partial s}+\dot{z}^{\mu}(s) A_{\mu}^{(R)}(z(s)) \mathrm{hol}^{(R)}=0
$$

where $\dot{z}^{\mu}(s)=\mathrm{d} z^{\mu}(s) / \mathrm{d} s$.

One can exponentiate the operator (4.3) to write

$$
\operatorname{hol}^{(R)}(\gamma)=\mathcal{P} \exp \left(\mathrm{i} \oint_{\gamma} A^{(R)}\right)
$$

The equivalence of the two formulas is provided by rewriting the argument of the exponential in terms of the pullback under the map (4.2) as

$$
\oint_{\gamma} A=\int_{[0,1]} \gamma^{*}(A)
$$

from which we may expand the path-ordered exponential (4.5) explicitly as

$$
\begin{array}{rl}
\operatorname{hol}^{(R)}(\gamma)=1+\sum_{n=1}^{\infty} \mathrm{i}^{n} \int_{0}^{1} \mathrm{~d} s_{1} \int_{0}^{s_{1}} & \mathrm{~d} s_{2} \cdots \int_{0}^{s_{n-1}} \mathrm{~d} s_{n} \dot{z}^{\mu_{1}}\left(s_{1}\right) \dot{z}^{\mu_{2}}\left(s_{2}\right) \cdots \dot{z}^{\mu_{n}}\left(s_{n}\right) \\
& \times A_{\mu_{1}}^{(R)}\left(z\left(s_{1}\right)\right) A_{\mu_{2}}^{(R)}\left(z\left(s_{2}\right)\right) \cdots A_{\mu_{n}}^{(R)}\left(z\left(s_{n}\right)\right) .
\end{array}
$$


Since the composition law of the group is not affected by the twist, and since the loop (4.2) is an embedding of the one-dimensional manifold $\mathbb{S}^{1}$ in $\mathcal{M}$, there are no star-products required in the definition of the holonomy operator. It thus transforms as usual under the ordinary adjoint action of the gauge group.

This is very natural from an algebraic point of view, as the gauge fields in (4.7) are generically multiplied together at separated points and the holonomy can thus be regarded as an element of the tensor algebra

$$
\operatorname{hol}^{(R)}(\gamma) \in \bigoplus_{n=0}^{\infty}\left(\mathcal{A} \otimes U\left(\mathfrak{g}^{(R)}\right)\right)^{\otimes n}
$$

As a consequence, there is no reason a priori to compose (4.8) with the deformed product $\mu: \mathcal{A} \otimes \mathcal{A} \rightarrow \mathcal{A}$. Since the holonomy operator is a generator element of the group algebra $\mathcal{H}=\mathbb{C} G \supset G$, it acts on tensor products of fields through the twisted coproduct as

$$
\Delta\left(\operatorname{hol}^{(R)}(\gamma)\right)=\mathcal{F}^{-1} \circ\left(\operatorname{hol}^{\left(R_{1}\right)}(\gamma) \otimes \operatorname{hol}^{\left(R_{2}\right)}(\gamma)\right) \circ \mathcal{F} .
$$

From the remarks made at the end of Section 2.2 it follows that $\operatorname{hol}^{(R)}(\gamma)$ is classically covariant under area-preserving diffeomorphisms, as well as being gauge covariant.

However, this is not the only definition of Wilson loop operators which leads to gauge invariant observables that are covariant under area-preserving diffeomorphisms. We can systematically deform the holonomy operators (4.7) into the star-holonomy operators defined by

$$
\begin{aligned}
\mathcal{P} \exp _{\star}\left(\mathrm{i} \oint_{\gamma} A^{(R)}\right) \doteq 1+\sum_{n=1}^{\infty} \mathrm{i}^{n} & \int_{0}^{1} \mathrm{~d} s_{1} \int_{0}^{s_{1}} \mathrm{~d} s_{2} \cdots \int_{0}^{s_{n-1}} \mathrm{~d} s_{n} \dot{z}^{\mu_{1}}\left(s_{1}\right) \dot{z}^{\mu_{2}}\left(s_{2}\right) \cdots \dot{z}^{\mu_{n}}\left(s_{n}\right) \\
& \times A_{\mu_{1}}^{(R)}\left(z\left(s_{1}\right)\right) \star A_{\mu_{2}}^{(R)}\left(z\left(s_{2}\right)\right) \star \cdots \star A_{\mu_{n}}^{(R)}\left(z\left(s_{n}\right)\right) \cdot
\end{aligned}
$$

This definition amounts to replacing the tensor product algebra in (4.8) with the braided tensor product algebra [4, 27, 47, as naturally imposed by non-cocommutativity of the twisted coproduct $\Delta$. From the general analysis of Section 2 it follows that these operators possess both twisted gauge covariance and twisted symplectic covariance. They are also invariant under the adjoint action of the star-gauge group [25, 44], which is defined to be the gauge group $G$ with its multiplication deformed by the star product, i.e. if $u_{1}, u_{2}: \mathcal{M} \rightarrow G$ are gauge transformations, then $u_{1} \circ u_{2} \doteq u_{1} \star u_{2}$. In contrast to the twist deformation, the star-product deformation limits the choice of gauge group and of representation $R$ [15, 20, 46], in the simplest settings to the unitary groups $G=\mathrm{U}(N)$ and to the $N$-dimensional fundamental representation. On the other hand, twisted gauge covariance of (4.10) generically requires the gauge connection $A_{\mu}$ to be $U(\mathfrak{g})$-valued [5] so that the star-holonomy is in general a non-generator element of the group algebra $\mathbb{C} G^{(R)}$.

The definition of the star-holonomy operator, given in (4.10) as a star-deformation of the ordinary gauge holonomy in (4.7), can be understood more fundamentally in the context of twist deformations as follows. In the argument of the path-ordered exponential (4.5) we can regard the contour integral as a bilinear map

$$
\nu_{0}=\oint: \Omega \mathcal{M} \otimes \mathcal{V}(\mathcal{M}, U(\mathfrak{g})) \longrightarrow U(\mathfrak{g})
$$

where $\mathcal{V}(\mathcal{M}, U(\mathfrak{g}))$ is the affine space of enveloping algebra valued connection one-forms on $\mathcal{M}$ and the linearity with respect to the first factor is defined in terms of the natural $\mathbb{Z}$-module 
structure on the loop space $\Omega \mathcal{M}$. All three of the linear spaces in (4.11) carry a natural action of the semi-direct product of the group of gauge transformations with the diffeomorphism group of $\mathcal{M}$. Then just as we defined the twisted product in (2.3), following the philosophy of [4] we can deform the map (4.11) by combining it with an action of the twist to get $\nu \doteq \nu_{0} \circ \mathcal{F}$. The twisted map $\nu$ satisfies the usual covariance condition $g \circ \nu=\nu \circ \Delta(g)$. This is a conceptually useful point of view for calculational purposes, as it allows one to naturally combine variations of the gauge connection $A_{\mu}$ with reparametrizations of the contour $\gamma$ (i.e. of the embedding $\left.z^{\mu}(s)\right)$ in an invariant way.

The star-holonomy operators obey a natural star-deformed version of the parallel transport equation (4.4) [1, 44]. The corresponding Wilson loop is denoted

$$
W_{\star}[\gamma, A] \doteq \operatorname{Tr} \mathcal{P} \exp _{\star}\left(\mathrm{i} \oint_{\gamma} A\right)
$$

where for brevity we drop the representation label from the notation. Although the ordinary holonomy operators $\operatorname{hol}^{(R)}(\gamma)$ yield the natural observables with respect to the twisted gauge symmetry, in the remainder of this paper we will focus on star-gauge invariant observables constructed from the star-Wilson loops (4.12). The distinction between the two classes of observables is similar to the distinction between deformed and undeformed products (or braided and unbraided tensor products) of fields at separated spacetime points [9, 27, 47]. In some instances, the incorporation of both types of observables reflects invariance of the noncommutative gauge theory under both twisted and star gauge symmetries, which has recently been argued to be a generic requirement for consistency of twisted gauge theories [28]. Detailed comparisons between the two types of gauge symmetries can be found in [10].

\subsection{Star-Gauge Invariant Correlators}

Noncommutative Yang-Mills theory on $\mathbb{R}^{2}$ is defined by the action functional

$$
S_{\mathrm{YM}}^{\star}[A]=\frac{1}{2} \int \mathrm{d}^{2} x \operatorname{Tr} f^{2}
$$

where $f=F_{A}=\partial_{1} A_{2}-\partial_{2} A_{1}-\mathrm{i} e\left(A_{1} \star A_{2}-A_{2} \star A_{1}\right)$ is the $U(\mathfrak{g})$-valued noncommutative field strength. As the action (4.13) is defined by the star product, it is natural to consider star-gauge invariant correlation functions of the form

$$
\int \mathcal{D} A \mathrm{e}^{-S_{\mathrm{YM}}^{\star}[A]} \mathcal{O}_{1}^{\star}(A) \cdots \mathcal{O}_{n}^{\star}(A)
$$

where the operators $\mathcal{O}_{i}^{\star}(A)$ include the star-Wilson loops (4.12). The ordinary, undeformed Yang-Mills action $S_{\mathrm{YM}}[A]$ is invariant under area-preserving diffeomorphisms of $\mathbb{R}^{2}$, because in two dimensions the field strength $f$ is a scalar field and hence any diffeomorphism which preserves the area form $\mathrm{d}^{2} x$ is a symmetry of the action. Furthermore, any Wilson loop, constructed from a holonomy operator hol ${ }^{(R)}(\gamma)$, is a homotopy invariant which depends only on the area enclosed by the loop $\gamma$, and not on the shape of $\gamma$. This classical symmetry extends to the quantum gauge theory [50], and in particular the functional integral measure $\mathcal{D} A$ is invariant under the corresponding transformations of the gauge connection $A$. From the general analysis of Section 2 (see remarks at the end of Section 2.2), it follows that the classical noncommutative gauge theory is thus invariant under twisted area-preserving diffeomorphisms. We will now study the 
behaviour of the correlators (4.14) under the twisted symplectic symmetry, and decide whether or not it is preserved after quantization.

To work out Ward identities in path integral quantization for this case, we proceed as in Section 3 to first determine whether or not the correlation functions exhibit anomalous breakdown of the classical symplectic symmetry. For this, we expand the gauge fields as $A_{\mu}=A_{\mu}^{a} T^{a}$ where $T^{a}$ are the generators of the gauge group with $\operatorname{Tr}\left(T^{a} T^{b}\right)=\delta^{a b}$, and couple them to external currents $J^{\mu}=J_{a}^{\mu} T^{a}$ through the pairing $(J, A) \doteq \int \mathrm{d}^{2} x J_{a}^{\mu} A_{\mu}^{a}$. Then the general arguments of Section 3 can be used to infer that the quantum effective action constructed through a Legendre transformation of the connected generating functional

$$
-\log \int \mathcal{D} A \mathrm{e}^{-S_{\mathrm{YM}}^{\star}[A]-(J, A)}
$$

is invariant under twisted area-preserving diffeomorphisms. A possible obstruction to this reasoning in the present case is the implicit presence of the Faddeev-Popov determinant in the path integral measure $\mathcal{D} A$. Since it is constructed from the Gauss' law constraint, it is built by means of star-products of the gauge fields and hence we need to show that it is twist invariant under a variation of the gauge field $A$ by an area-preserving diffeomorphism.

That this is indeed the case can be seen by expressing the determinant as a functional integral over anticommuting ghost fields $\bar{c}^{a}, c^{a}$ in the adjoint representation of the gauge group with the Lorentz gauge action

$$
\begin{aligned}
S_{\mathrm{gh}}^{\star}[\bar{c}, c, A]=\int \mathrm{d}^{2} x\left(\bar{c}^{a} \square c^{a}+e f^{a b h} \bar{c}^{a} \star A_{\mu}^{b} \star \partial^{\mu} c^{h}\right. & \\
& \left.-\mathrm{i} e d^{a b h} \bar{c}^{a} \star\left(A_{\mu}^{h} \star \partial^{\mu} c^{b}-\partial^{\mu} c^{b} \star A_{\mu}^{h}\right)\right),
\end{aligned}
$$

where $f^{a b h}$ are the structure constants of the Lie algebra $\mathfrak{g}$ and $d^{a b h}$ are invariant tensors for the adjoint action of $\mathfrak{g}$. The proof of twisted invariance of integrals over scalar densities given in Section 3.2 can now be applied to (4.16). We conclude that the twisted symplectic symmetry is non-anomalous in this case. Hence we need only further study the behaviour of the star-Wilson loops (4.12) under twisted area-preserving diffeomorphisms, implemented at the quantum level in (4.14).

\subsection{Twist Transformations of Quantum Star-Wilson Loops}

To analyse the twisted quantum symmetries of the operators (4.12), we shall use a proper path integral representation. For this, we introduce a pair of one-dimensional complex auxilliary fields $\bar{\zeta}_{i}(s)$ and $\zeta_{i}(s)$ on the loop $\gamma$ which transform respectively in the fundamental and antifundamental representations of the gauge group. Their propagator is given by

$$
\left\langle\bar{\zeta}_{i}\left(s_{1}\right) \zeta_{j}\left(s_{2}\right)\right\rangle_{\zeta} \doteq \int \mathcal{D} \bar{\zeta} \mathcal{D} \zeta \bar{\zeta}_{i}\left(s_{1}\right) \zeta_{j}\left(s_{2}\right) \exp \left(-\int_{0}^{1} \mathrm{~d} s \bar{\zeta}_{k}\left(s-0^{+}\right) \dot{\zeta}_{k}(s)\right)=\delta_{i j} \Theta\left(s_{1}-s_{2}\right)
$$

where $\Theta(s)$ is the Heaviside step function. The regularization indicated in the functional integral takes care of the ambiguous value of $\Theta(s)$ at $s=0$. The technical details of this regularization play no role below. They are analysed in [36] (see also [3] for an analysis in a slightly different context). 
By using the propagator (4.17) and Wick's theorem, we can unravel the path ordering operation $\mathcal{P}[36$ in (4.12) to write the star-Wilson loop operator as

$$
W_{\star}[\gamma, A]=\left\langle\bar{\zeta}_{k}(0) \exp _{\star}\left(\mathrm{i} \int_{0}^{1} \mathrm{~d} s \bar{\zeta}_{i}\left(s-0^{+}\right) A_{\mu}^{i j}(z(s)) \zeta_{j}(s) \dot{z}^{\mu}(s)\right) \zeta_{k}(1)\right\rangle_{\zeta} .
$$

We have dropped an irrelevant factor induced by the vacuum graphs of the auxilliary quantum field theory on $\gamma$ (which is equal to one for unimodular gauge groups and can be handled in exactly the same way as we do with (4.18) below). In (4.18) the star-products act only on the arguments of the gauge fields and do not involve the auxilliary fields. To avoid cluttered formulas below, we therefore introduce the shorthand notation

$$
\underline{A}_{\mu}(z(s)) \doteq \bar{\zeta}_{i}\left(s-0^{+}\right) A_{\mu}^{i j}(z(s)) \zeta_{j}(s) .
$$

These fields can be formally star multiplied in (4.18) without worrying about reordering each term, as our manipulations will not depend on the details of the loop integrals anyway.

We now expand the exponential in (4.18) and check for twisted symplectic invariance order by order in the loop embedding functions $z^{\mu}$. The zeroth and first order terms are trivially invariant as they do not involve any star products. The second order term is proportional to

$$
\int_{0}^{1} \mathrm{~d} s_{1} \int_{0}^{1} \mathrm{~d} s_{2} \underline{A}_{\mu}\left(z\left(s_{1}\right)\right) \star \underline{A}_{\nu}\left(z\left(s_{2}\right)\right) \dot{z}^{\mu}\left(s_{1}\right) \dot{z}^{\nu}\left(s_{2}\right) .
$$

Since there is no star product between $\underline{A}_{\mu}$ and $\dot{z}^{\mu}$, the arguments used in Section 2.2 do not guarantee the scalar property of this term and we must check its twisted transformation properties directly. Consider the variation

$$
\underline{A}_{\mu} \longmapsto \underline{A}_{\mu}+\delta_{\xi}^{\left(R_{1}\right)} \underline{A}_{\mu}
$$

of the abelianized gauge field (4.19) under an infinitesimal area-preserving diffeomorphism generated by a vector field $\xi$, where $R_{1}$ is the representation of the diffeomorphism group defined in (2.20). Discarding that part which can be absorbed into a deformation of the contour $\gamma$ under the diffeomorphism, one finds that the variation of (4.20) is given by

$$
\begin{aligned}
& \int_{0}^{1} \mathrm{~d} s_{1} \int_{0}^{1} \mathrm{~d} s_{2} \dot{z}^{\mu}\left(s_{1}\right) \dot{z}^{\nu}\left(s_{2}\right) \\
& \times \sum_{k=1}^{\infty} \frac{(-\mathrm{i} \theta / 2)^{k}}{k !} \sum_{l=0}^{k}\left(\begin{array}{c}
k \\
l
\end{array}\right)(-1)^{l}\left(\delta_{\partial_{1}^{k-l} \partial_{2}^{l} \xi}^{\left(R_{1}\right)} \underline{A}_{\mu}\left(z\left(s_{1}\right)\right) \star \partial_{2}^{k-l} \partial_{1}^{l} \underline{A}_{\nu}\left(z\left(s_{2}\right)\right)\right. \\
& \\
&\left.+(-1)^{k} \partial_{2}^{k-l} \partial_{1}^{l} \underline{A}_{\mu}\left(z\left(s_{1}\right)\right) \star \delta_{\partial_{1}^{k-l} \partial_{2}^{l} \xi}^{\left(R_{1}\right)} \underline{A}_{\nu}\left(z\left(s_{2}\right)\right)\right) .
\end{aligned}
$$

Each term of the summations in (4.22) is of the generic form

$$
\begin{aligned}
\int_{0}^{1} \mathrm{~d} s_{2} & \dot{z}^{\nu}\left(s_{2}\right) \int_{0}^{1} \mathrm{~d} s_{1} \dot{z}^{\mu}\left(s_{1}\right) \\
& \times\left(\partial_{1}^{k-l} \partial_{2}^{l} \xi^{\sigma} \partial_{\sigma} \underline{A}_{\mu}\left(z\left(s_{1}\right)\right)+\partial_{\mu} \partial_{1}^{k-l} \partial_{2}^{l} \xi^{\sigma} \underline{A}_{\sigma}\left(z\left(s_{1}\right)\right)\right) \star \partial_{2}^{k-l} \partial_{1}^{l} \underline{A}_{\nu}\left(z\left(s_{2}\right)\right) .
\end{aligned}
$$

At this point, it is tempting to use the twist deformation of the bilinear contour map (4.11) described in Section 4.1 above to absorb the variation of the contravariant vector field $\underline{A}_{\mu}$ 
into a deformation of the loop $\gamma$ (by appropriately redefining the embedding functions $z^{\mu}(s)$ ). But this would produce a sum of loop integrals, each one taken over a different contour. The only straightforward way to proceed is to restrict ourselves to some subgroup of the group of area-preserving diffeomorphisms and study its contribution to (4.22).

Let us expand the smooth vector field $\xi$ in a Taylor series

$$
\xi^{\mu}(x) \doteq \sum_{l=0}^{\infty} L_{\sigma_{1} \cdots \sigma_{l}} x^{\sigma_{1}} \cdots x^{\sigma_{l}}
$$

where $L^{\mu}{ }_{\sigma_{1} \cdots \sigma_{l}}$ is a Schwartz sequence of constant tensors which are completely symmetric in their lower indices. The divergence free constraint (3.15) is equivalent to the traceless conditions

$$
L_{\sigma_{1} \cdots \sigma_{l} \mu}^{\mu}=0
$$

The expression (4.22) may then be rewritten in the convenient form

$$
\begin{aligned}
& \int_{0}^{1} \mathrm{~d} s_{1} \int_{0}^{1} \mathrm{~d} s_{2} \dot{z}^{\mu}\left(s_{1}\right) \dot{z}^{\nu}\left(s_{2}\right) \sum_{l=0}^{\infty}\left(z^{\alpha_{1}}\left(s_{1}\right) \cdots z^{\alpha_{l}}\left(s_{1}\right) \sum_{k=1}^{\infty} \frac{\mathrm{i} k}{k !} \theta^{\sigma_{1} \rho_{1}} \cdots \theta^{\sigma_{k} \rho_{k}}\right. \\
& \times\left(L^{\rho}{ }_{\mu \sigma_{1} \cdots \sigma_{k} \alpha_{1} \cdots \alpha_{l}} \underline{A}_{\rho}\left(z\left(s_{1}\right)\right)+\delta^{\rho}{ }_{\mu} L^{\sigma}{ }_{\sigma_{1} \cdots \sigma_{k} \alpha_{1} \cdots \alpha_{l}} \partial_{\sigma} \underline{A}_{\rho}\left(z\left(s_{1}\right)\right)\right) \star \partial_{\rho_{1}} \cdots \partial_{\rho_{k}} \underline{A}_{\nu}\left(z\left(s_{2}\right)\right) \\
& \quad+\text { sym. })
\end{aligned}
$$

where the only non-vanishing components of the tensor $\theta^{\mu \nu}$ are $\theta^{12}=-\theta^{21}=\theta$, and the additional term is the corresponding symmetric contribution with $s_{1}$ and $s_{2}$ interchanged.

Let us first examine the expression (4.26) in the case of a unimodular linear affine transformation (2.25), i.e. a global area-preserving diffeomorphism in $\mathfrak{s l}(2, \mathbb{R}) \rtimes \mathbb{R}^{2}$. One obtains

$$
\begin{array}{r}
\int_{0}^{1} \mathrm{~d} s_{1} \int_{0}^{1} \mathrm{~d} s_{2} \dot{z}^{\mu}\left(s_{1}\right) \dot{z}^{\nu}\left(s_{2}\right) \mathrm{i} \theta^{\sigma_{1} \rho_{1}}\left(\delta^{\rho}{ }_{\mu} L^{\sigma} \sigma_{1} \partial_{\sigma} \underline{A}_{\rho}\left(z\left(s_{1}\right)\right) \star \partial_{\rho_{1}} \underline{A}_{\nu}\left(z\left(s_{2}\right)\right)\right. \\
\left.+\delta^{\rho}{ }_{\nu} L_{\rho_{1}}^{\sigma} \partial_{\sigma_{1}} \underline{A}_{\mu}\left(z\left(s_{1}\right)\right) \star \partial_{\sigma} \underline{A}_{\rho}\left(z\left(s_{2}\right)\right)\right) \\
=\int_{0}^{1} \mathrm{~d} s_{1} \int_{0}^{1} \mathrm{~d} s_{2} \dot{z}^{\mu}\left(s_{1}\right) \dot{z}^{\nu}\left(s_{2}\right) \mathrm{i} \theta^{\sigma_{1} \rho_{1}} \\
\left(L_{\sigma_{1}}^{\sigma} \delta_{\rho_{1}}^{\rho}+\delta_{\sigma_{1}}^{\sigma} L_{\rho_{1}}\right) \\
\times \partial_{\sigma} \underline{A}_{\mu}\left(z\left(s_{1}\right)\right) \star \partial_{\rho} \underline{A}_{\nu}\left(z\left(s_{2}\right)\right) .
\end{array}
$$

Using the traceless property (4.25) one sees that (4.27) vanishes. However, with a bit of work it can be seen that quadratic and higher-order terms in $x^{\mu}$ in the expansion (4.24) of the vector field $\xi$ do not vanish even with the traceless property (4.25) taken into account. The generalization of this calculation to arbitrarily high orders in the expansion of the exponential in (4.18) is straightforward.

We conclude that the star-Wilson loop is twist invariant only under global area-preserving diffeomorphims, similarly to the property that linear affine transformations are the only spacetime symmetries under which the Moyal product is covariant [29]. However, this does not mean that the star-Wilson loop is not invariant under general twisted symplectic transformations, but only that our attempt to realize twisted area-preserving diffeomorphisms as a symmetry of the noncommutative quantum field theory cannot be implemented on the star-Wilson loop in a 
way that keeps it invariant. This result explains the shape dependence and loss of non-linear symplectic invariance of the correlators of Wilson loops observed previously in [3, 13, 22, It also applies to any operator built from the star-Wilson loop, and it persists for open Wilson lines as our arguments hold as well when the contour $\gamma$ is not closed. Thus any star-gauge invariant observable will break the twisted symmetry under non-linear area-preserving maps. Since the effect of the twist acts on the contour $\gamma$ globally, we also see that the twist variation cannot be cancelled by means of any local operator insertion. The only remaining hope for full symplectic invariance is an alternative definition of the twisted noncommutative quantum gauge theory using the braided Wick expansions of [37, for example, which has recently been argued to be the correct framework for the implementation of twisted symmetries in noncommutative field theory [9].

\section{Acknowledgments}

We thank A.P. Balachandran, C. Sämann, H. Steinacker, S. Vaidya and J.D. Vergara for helpful discussions. This work was supported in part by the EU-RTN Network Grant MRTN-CT-2004005104. The work of M.R. was supported in part by a Fellowship of the Fondazione Angelo della Riccia.

\section{References}

[1] A.Y. Alekseev and A.G. Bytsko, "Wilson Lines on Noncommutative Tori", Phys. Lett. B 482 (2000) 271-275 hep-th/0002101.

[2] L. Alvarez-Gaume, F. Meyer and M.A. Vazquez-Mozo, "Comments on Noncommutative Gravity", Nucl. Phys. B 753 (2006) 92-117 hep-th/0605113.

[3] J. Ambjørn, A. Dubin and Y. Makeenko, "Wilson Loops in 2D Noncommutative Euclidean Gauge Theory I: Perturbative Expansion", J. High Energy Phys. 0407 (2004) 044 hep-th/0406187.

[4] P. Aschieri, M. Dimitrijevic, F. Meyer and J. Wess, "Noncommutative Geometry and Gravity", Class. Quant. Grav. 23 (2006) 1883-1912 hep-th/0510059.

[5] P. Aschieri, M. Dimitrijevic, F. Meyer, S. Schraml and J. Wess, "Twisted Gauge Theories", Lett. Math. Phys. 78 (2006) 61-71 hep-th/0603024.

[6] P. Aschieri, C. Blohmann, M. Dimitrijevic, F. Meyer, P. Schupp and J. Wess, "A Gravity Theory on Noncommutative Spaces", Class. Quant. Grav. 22 (2005) 3511-3532 hep-th/0504183.

[7] A.P. Balachandran, T.R. Govindarajan, K.S. Gupta and S. Kurkcuoglu, "Noncommutative Two-Dimensional Gravities", Class. Quant. Grav. 23 (2006) 5799-5810 hep-th/0602265.

[8] A.P. Balachandran, A. Pinzul, B.A. Qureshi, S. Vaidya and I.I.S. Bangalore, "Poincaré Invariant Gauge and Gravity Theories on the Grönewold-Moyal Plane", hep-th/0608138.

[9] A.P. Balachandran, T.R. Govindarajan, G. Mangano, A. Pinzul, B.A. Qureshi and S. Vaidya, "Statistics and UV/IR Mixing with Twisted Poincaré Invariance", hep-th/0608179.

[10] R. Banerjee and S. Samanta, "Gauge Generators, Transformations and Identities on a Noncommutative Space", hep-th/0608214; "Gauge Symmetries on $\theta$-Deformed Spaces", hep-th/0611249. 
[11] A. Bassetto and F. Vian, "Wilson Line Correlators in Two-Dimensional Noncommutative Yang-Mills Theory", J. High Energy Phys. 0210 (2002) 004 hep-th/0207222.

[12] A. Bassetto, G. Nardelli and A. Torrielli, "Perturbative Wilson Loop in TwoDimensional Noncommutative Yang-Mills Theory", Nucl. Phys. B 617 (2001) 308320 hep-th/0107147]; "Scaling Properties of the Perturbative Wilson Loop in TwoDimensional Noncommutative Yang-Mills Theory", Phys. Rev. D 66 (2002) 085012 hep-th/0205210.

[13] A. Bassetto, G. De Pol, A. Torrielli and F. Vian, "On the Invariance under Area-Preserving Diffeomorphisms of Noncommutative Yang-Mills Theory in Two Dimensions", J. High Energy Phys. 0505 (2005) 061 hep-th/0503175.

[14] W. Bietenholz, F. Hofheinz and J. Nishimura, "The Renormalizability of $2 D$ YangMills Theory on a Noncommutative Geometry", J. High Energy Phys. 0209 (2002) 009 hep-th/0203151.

[15] L. Bonora, M. Schnabl, M.M. Sheikh-Jabbari and A. Tomasiello, "Noncommutative SO $(N)$ and $\operatorname{Sp}(N)$ Gauge Theories", Nucl. Phys. B 589 (2000) 461-474 hep-th/0006091.

[16] X. Calmet, "Spacetime Symmetries of Noncommutative Spaces", Phys. Rev. D 71 (2005) 085012 hep-th/0411147].

[17] M. Chaichian and A. Tureanu, "Twist Symmetry and Gauge Invariance", Phys. Lett. B 637 (2006) 199-202 hep-th/0604025].

[18] M. Chaichian, A. Tureanu and G. Zet, "Twist as a Symmetry Principle and the Noncommutative Gauge Theory Formulation", hep-th/0607179.

[19] M. Chaichian, P.P. Kulish, K. Nishijima and A. Tureanu, "On a Lorentz-Invariant Interpretation of Noncommutative Spacetime and its Implications on Noncommutative QFT", Phys. Lett. B 604 (2004) 98-102 hep-th/0408069.

[20] M. Chaichian, P. Presnajder, M.M. Sheikh-Jabbari and A. Tureanu, "Noncommutative Gauge Field Theories: A No-Go Theorem", Phys. Lett. B 526 (2002) 132-136 arXiv:hep-th/0107037.

[21] V. Chari and A. Pressley, A Guide to Quantum Groups (Cambridge University Press, 1994).

[22] M. Cirafici, L. Griguolo, D. Seminara and R.J. Szabo, "Morita Duality and Noncommutative Wilson Loops in Two Dimensions", J. High Energy Phys. 0510 (2005) 030 hep-th/0506016.

[23] M. Dimitrijevic and J. Wess, "Deformed Bialgebra of Diffeomorphisms", hep-th/0411224.

[24] H. Dorn and A. Torrielli, "Loop Equation in Two-Dimensional Noncommutative Yang-Mills Theory", J. High Energy Phys. 0401 (2004) 026 [hep-th/0312047].

[25] M.R. Douglas and N.A. Nekrasov, "Noncommutative Field Theory", Rev. Mod. Phys. 73 (2001) 977-1029 hep-th/0106048.

[26] V.G. Drinfeld, "Quasi-Hopf Algebras”, Alg. Anal. 1N6 (1989) 114-148.

[27] G. Fiore and J. Wess, "On 'full' Twisted Poincaré Symmetry and QFT on Moyal-Weyl Spaces", hep-th/0701078.

[28] S. Giller, C. Gonera, P. Kosinski and P. Maslanka, "On the Consistency of Twisted Gauge Theory", hep-th/0701014.

[29] J.M. Gracia-Bondia, F. Lizzi, F.R. Ruiz and P. Vitale, "Noncommutative Spacetime Symmetries: Twist versus Covariance", Phys. Rev. D 74 (2006) 025014 hep-th/0604206. 
[30] L. Griguolo and D. Seminara, "Classical Solutions of the TEK Model and Noncommutative Instantons in Two Dimensions", J. High Energy Phys. 0403 (2004) 068 hep-th/0311041.

[31] L. Griguolo, D. Seminara and R.J. Szabo, "Instantons, Fluxons and Open Gauge String Theory", Adv. Theor. Math. Phys. 9 (2005) 775-860 hep-th/0411277].

[32] L. Griguolo, D. Seminara and P. Valtancoli, "Towards the Solution of Noncommutative $\mathrm{YM}_{2}$ : Morita Equivalence and Large $N$ Limit", J. High Energy Phys. 0112 (2001) 024 hep-th/0110293.

[33] H. Grosse, J. Madore and H. Steinacker, "Field Theory on the $q$-Deformed Fuzzy Sphere II: Quantization", J. Geom. Phys. 43 (2002) 205-240 hep-th/0103164.

[34] F. Lizzi, R.J. Szabo and A. Zampini, "Geometry of the Gauge Algebra in Noncommutative Yang-Mills Theory", J. High Energy Phys. 0108 (2001) 032 hep-th/0107115.

[35] F. Lizzi, S. Vaidya and P. Vitale, "Twisted Conformal Symmetry in Noncommutative TwoDimensional Quantum Field Theory", Phys. Rev. D 73 (2006) 125020 hep-th/0601056.

[36] N.E. Mavromatos and R.J. Szabo, "Matrix D-Brane Dynamics, Logarithmic Operators and Quantization of Noncommutative Spacetime", Phys. Rev. D 59 (1999) 104018 hep-th/9808124.

[37] R. Oeckl, "Braided Quantum Field Theory", Commun. Math. Phys. 217 (2001) 451-473 hep-th/9906225.

[38] R. Oeckl, "Untwisting Noncommutative $\mathbb{R}^{d}$ and the Equivalence of Quantum Field theories", Nucl. Phys. B 581 (2000) 559-574 hep-th/0003018.

[39] L.D. Paniak and R.J. Szabo, "Instanton Expansion of Noncommutative Gauge Theory in Two Dimensions", Commun. Math. Phys. 243 (2003) 343-387 hep-th/0203166.

[40] L.D. Paniak and R.J. Szabo, "Open Wilson Lines and Group Theory of Noncommutative Yang-Mills Theory in Two Dimensions", J. High Energy Phys. 0305 (2003) 029 hep-th/0302162.

[41] L.D. Paniak and R.J. Szabo, "Lectures on Two-Dimensional Noncommutative Gauge Theory 2: Quantization", Lect. Notes Phys. 662 (2005) 205-237 hep-th/0304268.

[42] N. Reshetikhin, "Multiparameter Quantum Groups and Twisted Quasitriangular Hopf Algebras", Lett. Math. Phys. 20 (1990) 331-335.

[43] M. Rosenbaum, J.D. Vergara and L.R. Juarez, "Canonical Quantization, Spacetime Noncommutativity and Deformed Symmetries in Field Theory", hep-th/0611160.

[44] R.J. Szabo, "Quantum Field Theory on Noncommutative Spaces", Phys. Rept. 378 (2003) 207-299 hep-th/0109162.

[45] R.J. Szabo, "Symmetry, Gravity and Noncommutativity", Class. Quant. Grav. 23 (2006) R199-R242 hep-th/0606233.

[46] S. Terashima, "A Note on Superfields and Noncommutative Geometry", Phys. Lett. B 482 (2000) 276-282 hep-th/0002119.

[47] A. Tureanu, "Twist and Spin-Statistics Relation in Noncommutative Quantum Field Theory", Phys. Lett. B 638 (2006) 296-301 hep-th/0603219].

[48] D.V. Vassilevich, "Twist to Close", Mod. Phys. Lett. A 21 (2006) 1279-1284 hep-th/0602185. 
[49] J. Wess, "Deformed Coordinate Spaces: Derivatives", in: Mathematical, Theoretical and Phenomenological Challenges Beyond the Standard Model, Vrnjacka Banja (2003), pp. 122128 hep-th/0408080.

[50] E. Witten, "On Quantum Gauge Theories in Two Dimensions", Commun. Math. Phys. 141 (1991) 153-209.

[51] J. Zahn, "Remarks on Twisted Noncommutative Quantum Field Theory", Phys. Rev. D 73 (2006) 105005 hep-th/0603231. 